\title{
Alkyl-aryl-vancomycins: Multimodal glycopeptides with weak dependence on bacterial metabolic state
}

Paramita Sarkar, ${ }^{\mathrm{a}}$ Debajyoti Basak, ${ }^{\mathrm{a}}$ Riya Mukherjee, ${ }^{\mathrm{a}}$ Julia E. Bandow, ${ }^{\mathrm{b}}$ Jayanta Haldar ${ }^{\mathrm{a}}$

aAntimicrobial Research Laboratory, New Chemistry Unit and School of Advanced Materials, Jawaharlal Nehru Centre for Advanced Scientific Research (JNCASR), Jakkur, Bengaluru 560064, Karnataka, India

${ }^{\mathrm{b}}$ Applied Microbiology, Faculty of Biology and Biotechnology, RuhrUniversity Bochum Universitätsstraße 150, 44780 Bochum, Germany

*Corresponding author: Jayanta Haldar, Email ID: jayanta@jncasr.ac.in, Ph. No.: +91 802208 2565. 


\section{Table of Contents}

Results- Page S2

Characterisation of lead compound AAV-qC10- Page S5

\section{Results}

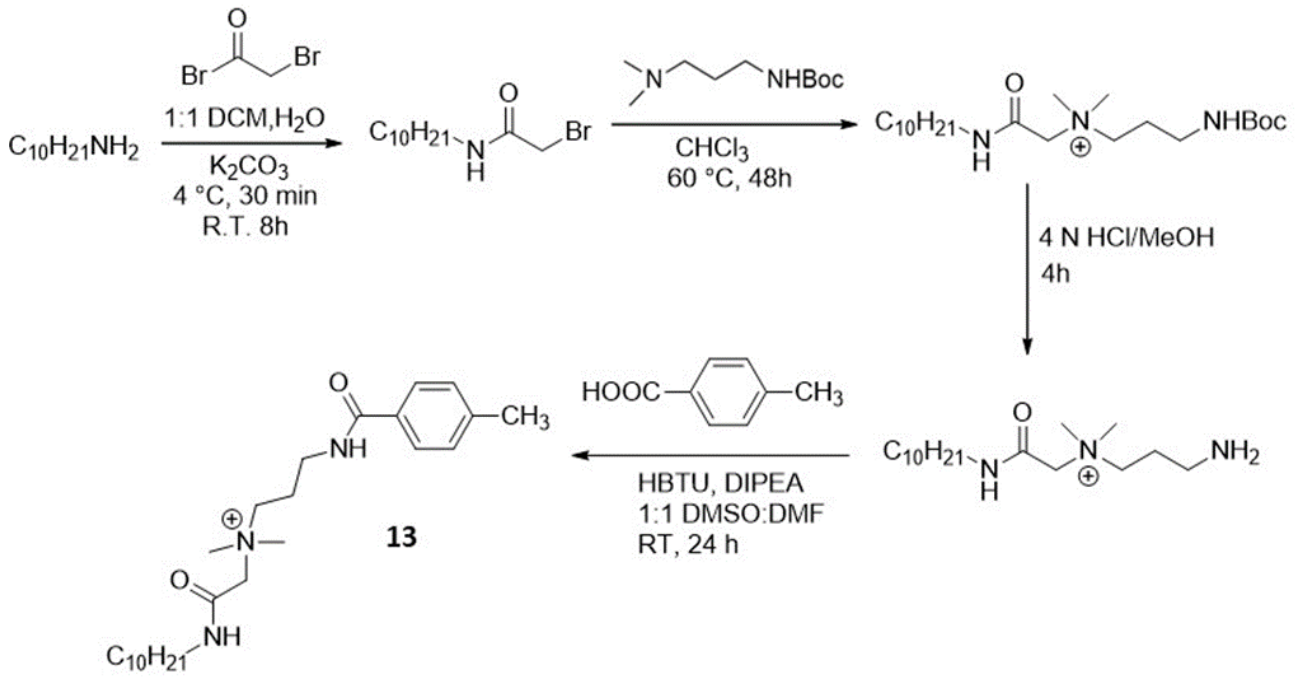

Scheme S1: Synthesis of control compound 13

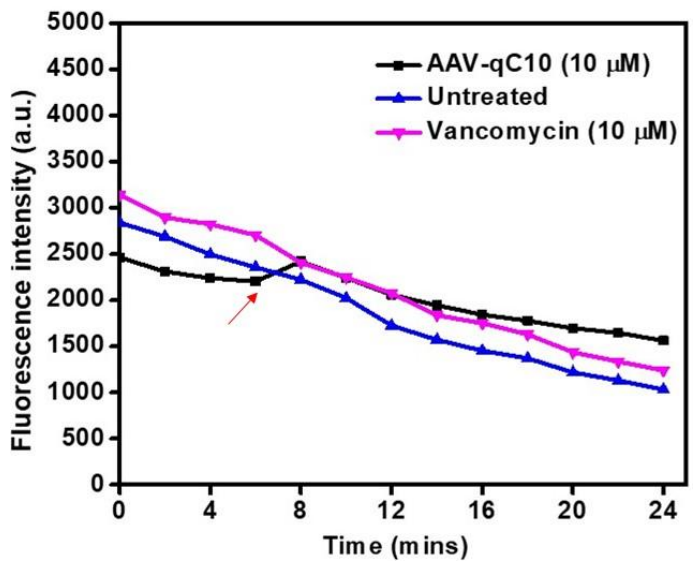

Figure $\mathrm{S} 1$. Kinetics of effect of vancomycin and $\mathrm{AAV}-\mathrm{qC} 10$ on fluorescence of $\mathrm{DisC}_{3}(5)$ dye. Red arrow indicates compound addition.

The gradual decrease in fluorescence of the dye results from the interaction with the polystyrene-surface of the microtiter plate (as mentioned in Ref 47) 


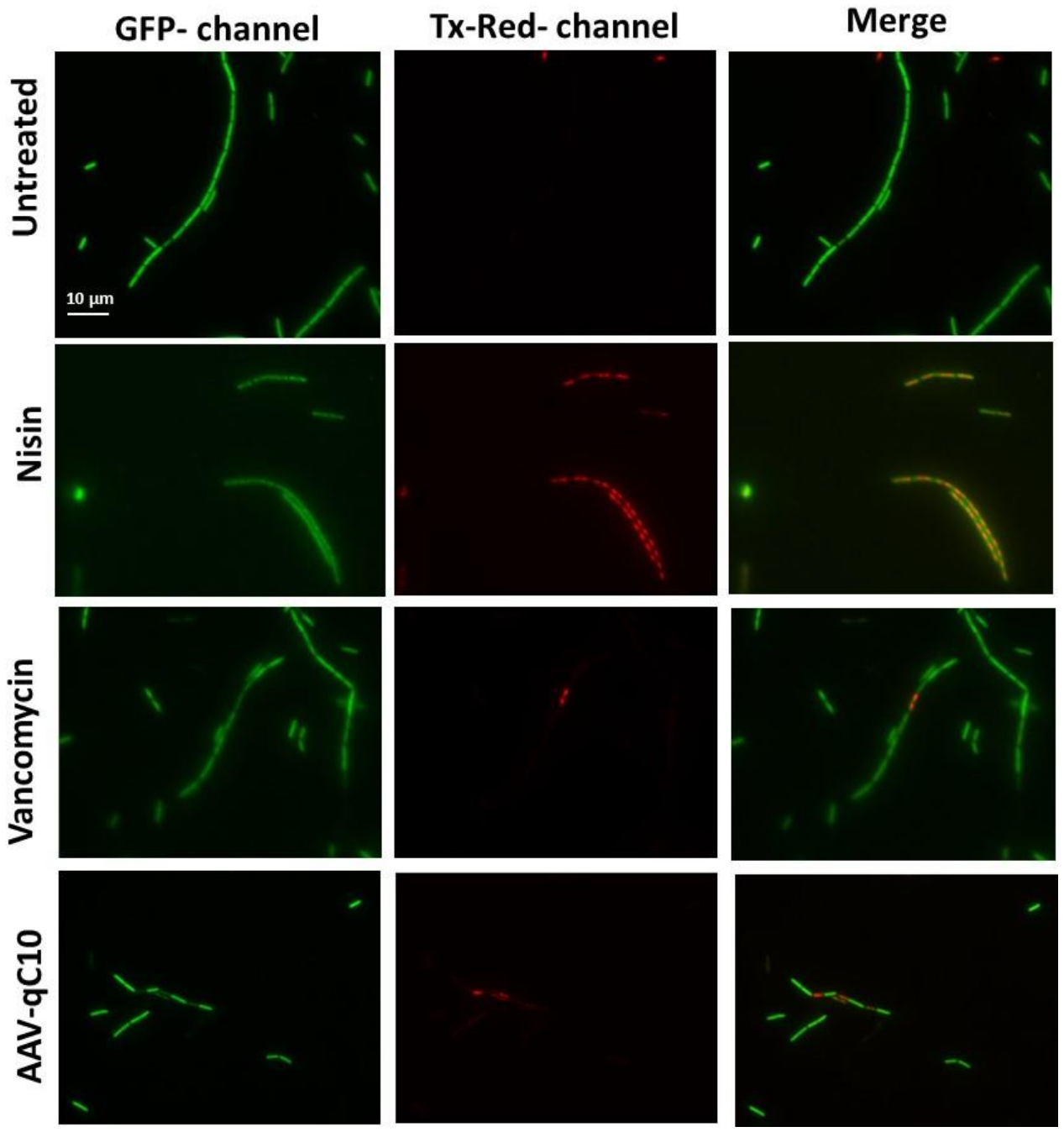

Figure S2. Fluorescence microscopy imaging to test for pore-formation as a mechanism of action of compounds through BAC-Light kit. Bacteria were treated with Nisin $(0.75 \mu \mathrm{g} / \mathrm{mL})$, vancomycin (PEC, $0.4 \mu \mathrm{M})$ and AAV-qC10 $(0.06 \mu \mathrm{M})$ and stained with SYTO9/PI for visualisation under the microscope. Scale bar corresponds to $10 \mu \mathrm{m}$ for all the images. 

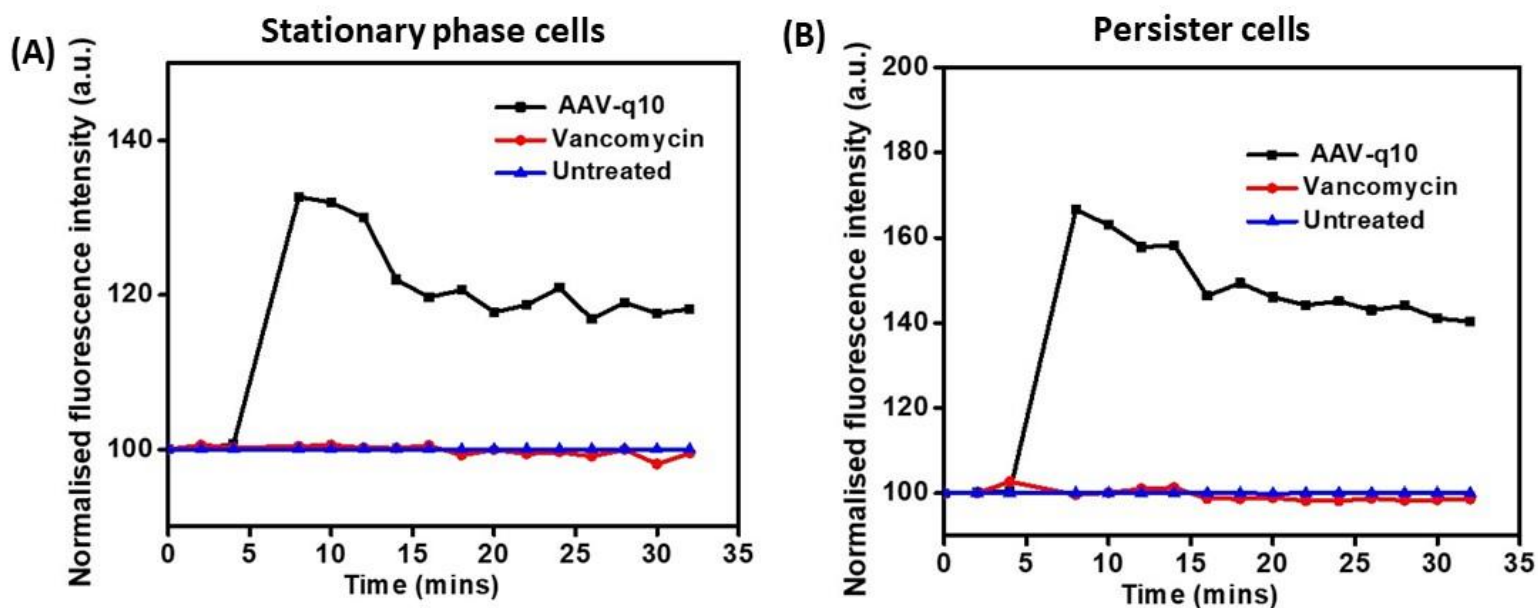

(C)

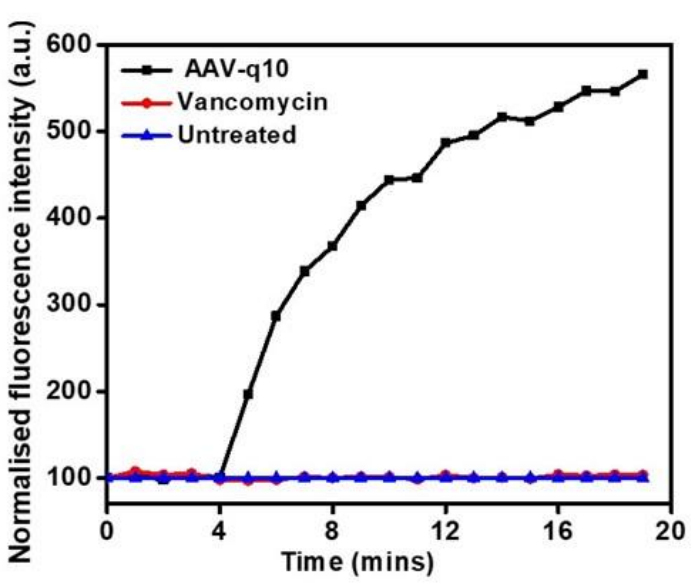

(D)

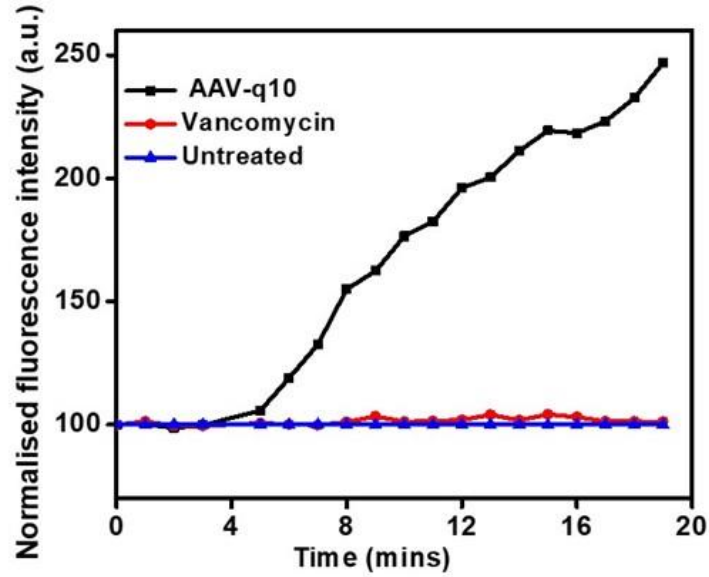

Figure S3. (A and B) Membrane depolarisation, (C and D) Membrane permeabilization of AAV-qC10 against stationary phase (A and C) and persister cells (A and D) of MRSA, post treatment with AAV-qC10 and vancomycin at $20 \mu \mathrm{M}$.

Table S1: Stability of AAV-qC10 in mouse plasma and liver homogenate through testing of MIC against MRSA and VRE post-incubation

\begin{tabular}{llll}
\hline & \multicolumn{3}{l}{ MIC of AAV-qC10 post incubation in $(\boldsymbol{\mu M})$} \\
\cline { 2 - 4 } Bacteria & Plasma & Liver homogenate & Media \\
\hline VRE 909 & 0.9 & 0.9 & 0.9 \\
MRSA ATCC 33591 & 0.9 & 0.9 & 0.9 \\
\hline
\end{tabular}




\section{Characterisation of AAV-qC10:}

HR-MS

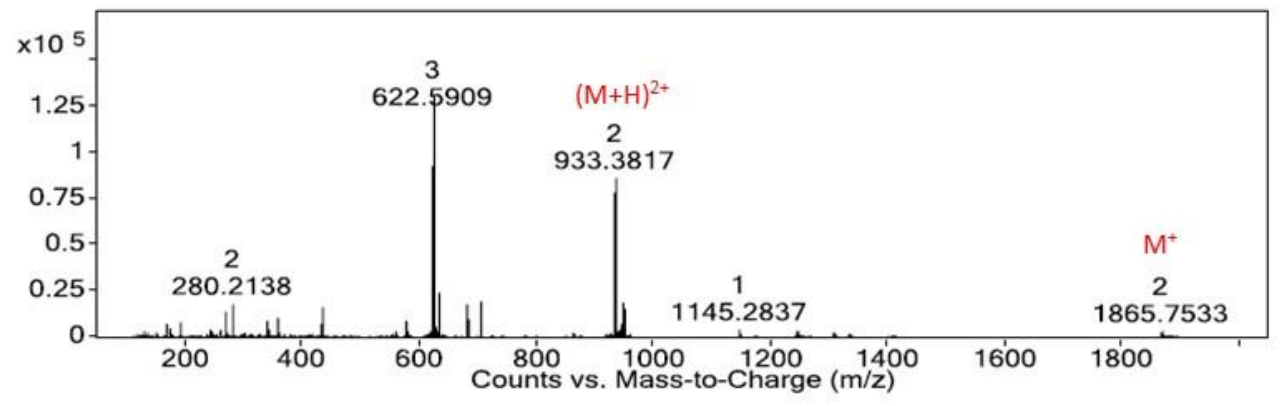

${ }^{1} \mathrm{H}-\mathrm{NMR}$

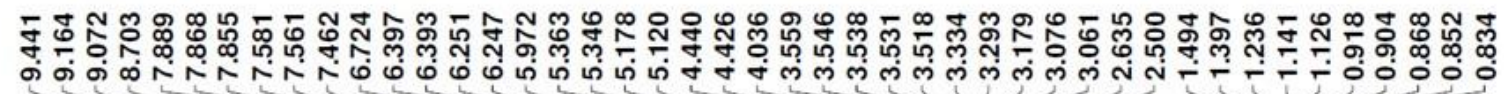

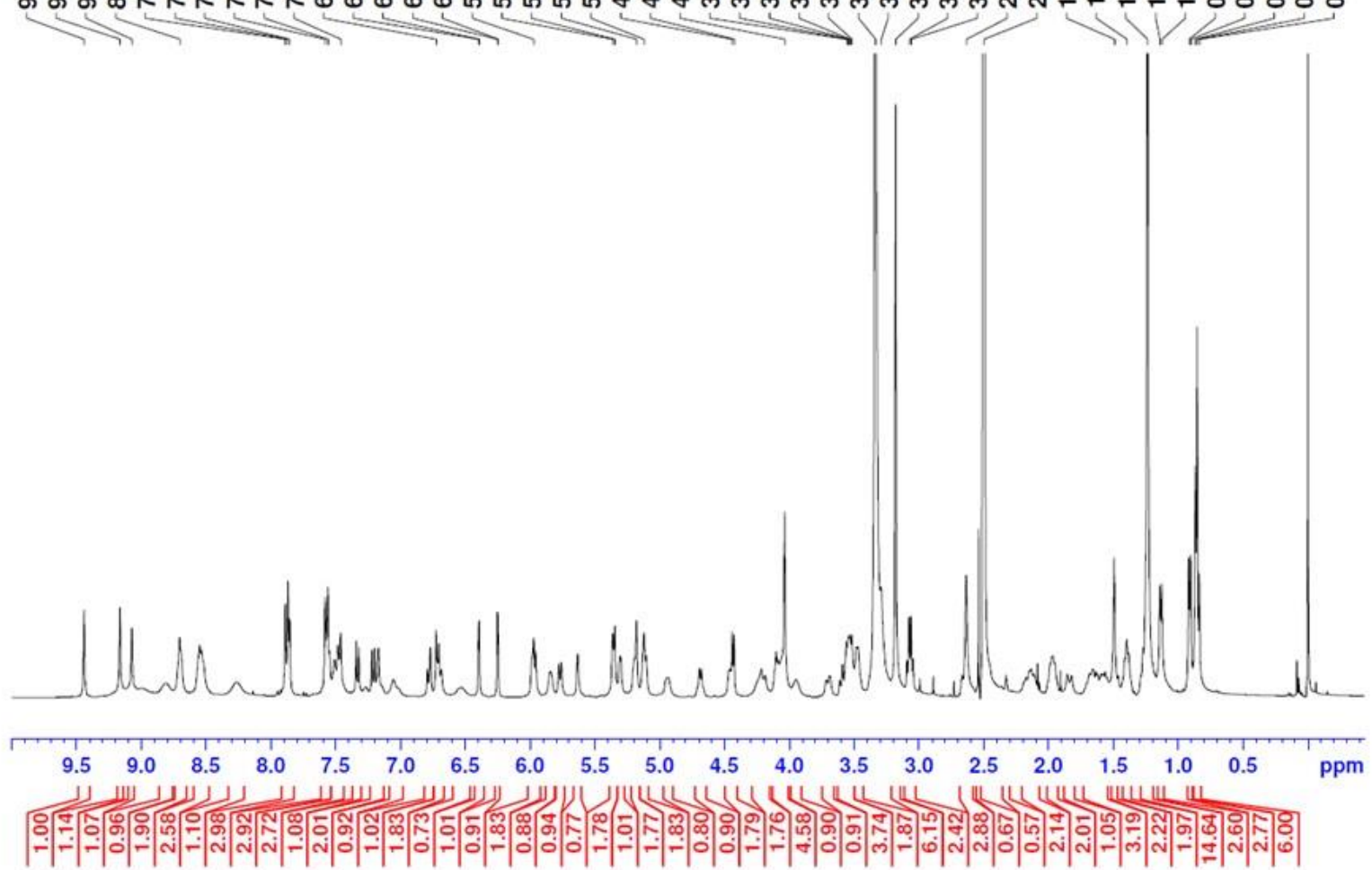

HPLC chromatogram:

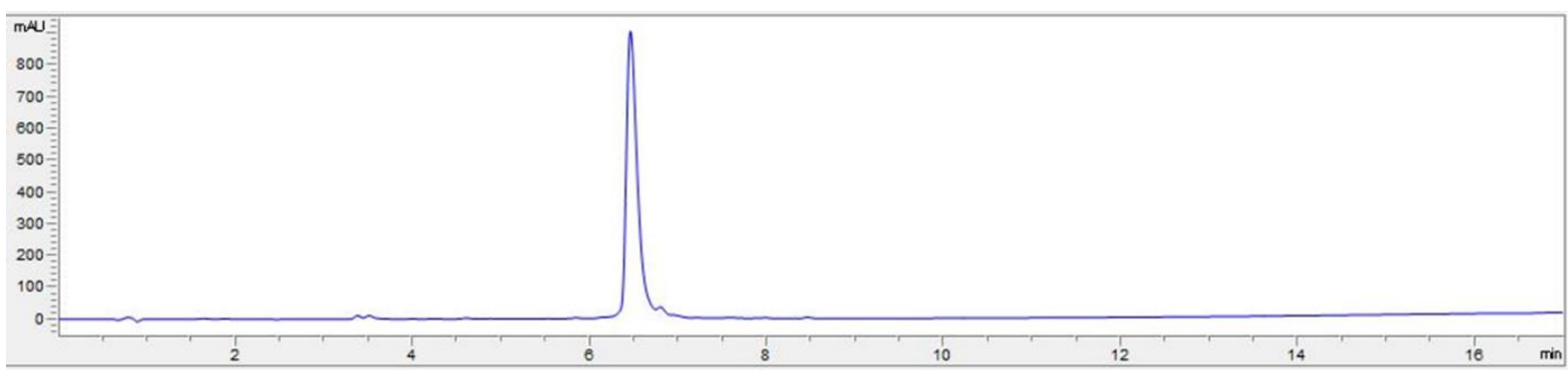

